Gut and Liver, Vol. 11, No. 3, May 2017, pp. 315-316

\title{
Clinical Considerations of Silent Gastroesophageal Reflux Disease in Morbidly Obese Patients
}

\author{
Sung Eun Kim \\ Department of Internal Medicine, Kosin University College of Medicine, Busan, Korea
}

See "Decreased Esophageal Sensitivity to Acid in Morbidly Obese Patients: A Cause for Concern?" by Vicente Ortiz, et al. on page 358, Vol. 11. No. 3, 2017

Obesity is regarded as a major cause of morbidity and mortality and threatens public health, resulting in social and economic problems. Therefore, obesity has become one of the most important diseases worldwide. ${ }^{1}$ The general definition of obesity is a body mass index (BMI) $\geq 30 \mathrm{~kg} / \mathrm{m}^{2}$. Patients with more severe obesity, such as a BMI $\geq 40 \mathrm{~kg} / \mathrm{m}^{2}$, are defined as morbidly obese (M0). ${ }^{2}$ Unfortunately, the prevalence of both obesity and MO has been steadily rising since 2000, and about 15.5 million adults in the United States were MO in 2010. ${ }^{2}$ Gastroesophageal reflux disease (GERD) is a common global gastrointestinal disease and is defined as a condition that occurs when the reflux of stomach contents triggers troublesome symptoms and/ or complications. The prevalence of GERD has been increasing with that of obesity, and several studies have evaluated the association between obesity and GERD.

A large retrospective study was performed on patients with GERD symptoms $(n=1,659)$ using 24 -hour esophageal $\mathrm{pH}$ monitoring and reported that increasing BMI was positively associated with increasing esophageal acid exposure and increasing lower esophageal sphincter (LES) defection $(\mathrm{p}<0.001)$. $^{3}$ El-Serag et $a{ }^{4}{ }^{4}$ also showed that acid reflux episodes, long reflux episodes ( $>5 \mathrm{~min}$ ), time with $\mathrm{pH}<4$, and calculated summary score in 24-hour esophageal $\mathrm{pH}$ monitoring were significantly higher in obese patients compared with patients with BMI $<25 \mathrm{~kg} / \mathrm{m}^{2}$. According to studies about the pathogenesis of GERD in obesity, several mechanisms, such as visceral fat at the gastroesophageal junction (GEJ), increased circulating hormones (e.g., estrogen), increased gastric pressure caused by the pressure within the intra-abdominal organ, disruption of the GEJ, and increased transient LES have been suggested to elucidate the association between GERD and obesity. ${ }^{5}$ However, research on GERD in MO patients, especially esophageal sensitivity in MO patients, has rarely been conducted, and the relationship between MO and GERD is still controversial. For that reason, this article has academic value in the comprehension of the pathogenesis of GERD in MO.

In this issue of Gut and Liver, Ortiz et al. ${ }^{6}$ performed a crosssectional study that investigated the esophageal sensitivity to acid in MO patients compared with non-MO controls with BMI $\leq 35 \mathrm{~kg} / \mathrm{m}^{2}$, and both groups were assumed to have abnormal esophageal acid exposure. A total of 58 patients were enrolled initially, comprised of $30 \mathrm{MO}$ and 28 non-MO patients. Among them, 14 MO and 28 non-MO were diagnosed with abnormal esophageal acid exposure using gastroscopy and/or 24-hour esophageal pH monitoring, and Bernstein test (0.1 M hydrochloric acid solution) was conducted in 42 patients for verifying esophageal symptoms and esophageal sensitivity. According to the results, MO patients experienced significantly fewer esophageal symptoms (14\% vs 96\%; odds ratio [OR], 0.006; 95\% confidence interval [CI], 0.001 to 0.075$)$ and decreased esophageal sensitivity (57\% vs 14\%; OR, 8; 95\% CI, 1.79 to 35.74) than non-MO patients. In addition, there was no significant difference in other demographic and epidemiologic factors in MO patients with or without abnormal esophageal acid exposure $(p=0.707)$. Therefore, the authors concluded that the silent GERD prevalent in MO patients may be due to decreased esophageal sensitivity.

This study revealed the importance of recognizing that silent GERD is common in MO patients and demonstrated decreased esophageal sensitivity in MO patients based on the Bernstein

Correspondence to: Sung Eun Kim

Department of Internal Medicine, Kosin University College of Medicine, 262 Gamcheon-ro, Seo-gu, Busan 49267, Korea

Tel: +82-51-990-5205, Fax: +82-51-990-5055, E-mail: solefide@hanmail.net

pISSN 1976-2283 eISSN 2005-1212 https://doi.org/10.5009/gnl17084

@) This is an Open Access article distributed under the terms of the Creative Commons Attribution Non-Commercial License (http://creativecommons.org/licenses/by-nc/4.0) which permits unrestricted non-commercial use, distribution, and reproduction in any medium, provided the original work is properly cited. 
test. Many physicians do not typically perform or delay diagnostic examinations in patients without symptoms. In this condition, MO patients without GERD symptoms could experience delayed discovery of erosive esophagitis, Barrett's esophagus, or even esophageal carcinoma. Unfortunately, a Mendelian randomization study verified that esophageal adenocarcinoma risk increased by 16\% (OR, 1.16; 95\% CI, 1.01 to 1.33) and Barrett's esophagus risk enhanced by $12 \%$ (OR, 1.12 ; 95\% CI, 1.00 to 1.25) per $1 \mathrm{~kg} / \mathrm{m}^{2}$ increase in BMI, and there was a statistically significant association between esophageal adenocarcinoma or Barrett's esophagus and BMI. ${ }^{7}$ Thus, obesity is independently related with esophageal adenocarcinoma and Barrett's esophagus, and MO patients should undergo more active diagnostic evaluations to identify esophageal disease.

With regard to esophageal symptoms of MO, a recent study in Spain revealed that about 45\% of asymptomatic or returning normal endoscopy MO patients had positive esophageal $\mathrm{pH}$ monitoring tests, and the authors suggested that MO without GERD does not rule out the existence of abnormal esophageal functional disorders. ${ }^{8}$ Mora et al. ${ }^{9}$ also demonstrated that 38 of 120 MO patients with abnormal 24-hour esophageal pH monitoring had no GERD symptoms. However, in $101 \mathrm{MO}$ patients with normal 24-hour esophageal $\mathrm{pH}$ results, 36 complained of GERD symptoms. In their study, the sensitivity and specificity of GERD symptoms in MO patients were 68.3\% (82/120) and 64.3\% (65/101), respectively. ${ }^{9}$ Another recent study using endoscopy reported that endoscopic findings were not associated with the presence of GERD or dyspepsia symptoms in MO patients, and the sensitivity and specificity of esophageal symptoms in MO patients were 44.2\% (27/61) and 50.6\% (41/81), respectively. ${ }^{10}$ These results show that the sensitivity and specificity of GERD symptoms in MO patients are very low, and it is suspected that there might be other unknown factors that influence the pathogenesis of GERD in MO patients compared to the pathogenesis of GERD in patients with obesity.

There are several limitations in this study. The sample size is small, and the control group is not composed of healthy individuals but those with a BMI $\leq 35 \mathrm{~kg} / \mathrm{m}^{2}$. Therefore, some individuals in the control group were obesity patients. Nevertheless, Ortiz et al. ${ }^{6}$ conducted a study using a different approach such as the Bernstein test and revealed useful information about the esophageal symptoms and esophageal sensitivity in MO patients. Further large-scale prospective studies are needed to es- tablish the pathogenesis of GERD in MO patients separate from obesity patients.

\section{CONFLICTS OF INTEREST}

No potential conflict of interest relevant to this article was reported.

\section{REFERENCES}

1. Hedley AA, Ogden CL, Johnson CL, Carroll MD, Curtin LR, Flegal KM. Prevalence of overweight and obesity among US children, adolescents, and adults, 1999-2002. JAMA 2004;291:2847-2850.

2. Sturm R, Hattori A. Morbid obesity rates continue to rise rapidly in the United States. Int J Obes (Lond) 2013;37:889-891.

3. Ayazi S, Hagen JA, Chan LS, et al. Obesity and gastroesophageal reflux: quantifying the association between body mass index, esophageal acid exposure, and lower esophageal sphincter status in a large series of patients with reflux symptoms. J Gastrointest Surg 2009;13:1440-1447.

4. El-Serag HB, Ergun GA, Pandolfino J, Fitzgerald S, Tran T, Kramer JR. Obesity increases oesophageal acid exposure. Gut 2007;56: 749-755.

5. Mion F, Dargent J. Gastro-oesophageal reflux disease and obesity: pathogenesis and response to treatment. Best Pract Res Clin Gastroenterol 2014;28:611-622.

6. Ortiz V, Alvarez-Sotomayor D, Sáez-González E, et al. Decreased esophageal sensitivity to acid in morbidly obese patients: a cause for concern? Gut Liver 2017;11:358-362.

7. Thrift AP, Shaheen NJ, Gammon MD, et al. Obesity and risk of esophageal adenocarcinoma and Barrett's esophagus: a Mendelian randomization study. J Natl Cancer Inst 2014;106:dju252.

8. Martín-Pérez J, Arteaga-González I, Martín-Malagón A, Díaz-Luis H, Casanova-Trujillo C, Carrillo-Pallarés AA. Frequency of abnormal esophageal acid exposure in patients eligible for bariatric surgery. Surg Obes Relat Dis 2014;10:1176-1180.

9. Mora F, Cassinello N, Mora M, Bosca M, Minguez M, Ortega J. Esophageal abnormalities in morbidly obese adult patients. Surg Obes Relat Dis 2016;12:622-628.

10. Carabotti M, Avallone M, Cereatti F, et al. Usefulness of upper gastrointestinal symptoms as a driver to prescribe gastroscopy in obese patients candidate to bariatric surgery: a prospective study. Obes Surg 2016;26:1075-1080. 